\title{
Decreasing procedure times with a standardized approach to ELVO cases
}

\author{
Ryan A McTaggart, ${ }^{1}$ Shadi Yaghi, $^{2}$ Grayson Baird, ${ }^{1}$ Richard A Haas, ${ }^{1,3}$ \\ Mahesh $V$ Jayaraman ${ }^{1,2,3}$
}

${ }^{1}$ Department of Diagnostic Imaging, Warren Alpert School of Medicine at Brown University, Rhode Island Hospital, Providence, Rhode Island, USA

${ }^{2}$ Department of Neurology, Warren Alpert School of Medicine at Brown University, Rhode Island Hospital,

Providence, Rhode Island, USA ${ }^{3}$ Department of Neurosurgery, Warren Alpert School of Medicine at Brown University, Rhode Island Hospital, Providence, Rhode Island, USA

\section{Correspondence to} Dr Mahesh V Jayaraman, Department of Diagnostic Imaging, Warren Alpert School of Medicine at Brown University, Rhode Island Hospital, 593 Eddy Street, Room 377, Providence, RI 02903 USA;

mjayaraman@lifespan.org

Received 13 May 2016 Revised 16 June 2016 Accepted 20 June 2016 Published Online First 8 July 2016

\section{ABSTRACT}

Background Embolectomy is the standard of care for emergent large vessel occlusion (ELVO), and needs to be done as quickly as possible for the best possible outcomes. Optimization of workflow and process is certainly paramount. One aspect of this is process improvement to standardize as much as possible the procedure in order to decrease variability among operators, which breeds familiarity for the entire team. Objective To evaluate the impact of a standardized approach to ELVO cases in decreasing times from groin puncture to first deployment of a stent-retriever and final recanalization.

Methods A retrospective review of 83 consecutive patients consisting of a pre-standardization phase (group 1) and those after standardization (group 2). The standardization process involved all three neurointerventional radiologists agreeing on a standard approach to the cases and to the equipment to be used. Times from groin puncture to first deployment of the stent-retriever and from puncture to final reperfusion were evaluated. Angiographic outcomes were scored using the Modified Thrombolysis in Cerebral Ischemia (mTICl) score. Complications from intracranial catheter manipulation (such as wire perforation) were also recorded. Clinical outcomes were assessed based on admission and discharge National Institute of Health Stroke Scale score.

Results There were 22 patients in group 1 and 61 patients in group 2. Mean times from groin puncture to first deployment were 39.8 min in group 1 and 20 min in group 2, a difference which was statistically significant $(p<0.0001)$. Overall times from puncture to final recanalization were reduced from 68.2 to $37 \mathrm{~min}$, also a statistically significant difference $(p<0.001)$. There were no cases of intraprocedural complications such as wire perforation or subarachnoid hemorrhage.

Conclusions A standardized approach to the equipment used and process for ELVO cases at a single institution can dramatically reduce procedure times.

\section{INTRODUCTION}

With the arrival of five positive trials, embolectomy is now the standard of care (in addition to IV tissue plasminogen activator (tPA)) for patients with emergent large vessel occlusion (ELVO) in the anterior circulation presenting within 6 hours from symptom onset. $^{1-5}$

Among the primary reasons these trials were positive were better endovascular tools, better pretreatment imaging, and an emphasis on rapid treatment. For example, in the ESCAPE trial the goal was from first image from non-contrast CT scan to groin puncture in $60 \mathrm{~min}$ or less. Similarly, the SWIFT-PRIME trial set a goal of CT angiography (CTA) images to groin puncture in $90 \mathrm{~min}$ or less (ideally $60 \mathrm{~min}$ or less). But what about once the procedure has begun? Median times from puncture to recanalization among the five trials ranged from $86 \mathrm{~min}$ (REVASCAT) to $33 \mathrm{~min}$ (SWIFT-PRIME).

We know there is a strong relationship between time from onset to recanalization and favorable outcome, dating back to the Interventional Management of Stroke (IMS) I and II trials. ${ }^{6}$ This has also held true in the modern embolectomy era. ${ }^{7}$ Workflow optimization to ensure rapid triage of patients with severe stroke and efficient in-hospital processes are vital to optimize time to angiography. 89 Our aim was to standardize what happened intraprocedurally once the patient arrived in the angiography suite and to examine how this impacted time from groin puncture to first deployment and recanalization.

Standardization of the workflow should reduce the number of steps needed in the staging process and reduce the number of decisions being made by the neurointerventional radiologist and other staff. In theory, these efforts should reduce both time and cognitive load (ie, amount of mental effort used in the working memory). ${ }^{10}$ Therefore, it is hypothesized that implementation of the standardization process should significantly decrease the time between initial groin puncture and recanalization.

\section{METHODS}

Institutional review board approval was obtained for a retrospective review of all patients treated by mechanical thrombectomy at a single comprehensive stroke center between 1 November 2014 and 1 January 2016. The starting date was chosen based on when tracking of the time from groin puncture to first deployment of stent-retriever (or first pass with direct aspiration) was started. We included all patients with an intracranial large vessel occlusion. Patients who underwent diagnostic angiography alone and those with more distal occlusions were excluded. Manuscript preparation began on 11 January 2016 and all patients who were treated and discharged during this time frame were included for analysis. All patients were treated with conscious sedation except those who had been intubated previously for airway concerns. We did not electively intubate any patients. 
The patients were divided into two groups: group 1 (prestandardization) refers to a cohort of patients during a period of time where several aspects of the case were at the discretion of the treating neurointerventionalist. These steps are described in more detail below.

\section{Standardization process}

The three neurointerventionalists (RAM, RAH, MVJ) who would be performing all the procedures met as a group to standardize the steps involved in the case. We agreed on answers to most of the aforementioned questions, with answers listed below. There were a total of three decisions which we agreed would have to be determined on a case-by-case basis (numbers 1, 4, and 12 below). However, the answers to these three steps can be determined from the pretreatment CTA, minimizing the need to change 'on the fly' whenever possible. Our consensus answers are listed below:

1. What size sheath should I place in the groin? $8 \mathrm{Fr}, 45 \mathrm{~cm}$ if there is concern about aortic arch tortuosity from CTA, otherwise $6 \mathrm{Fr}, 90 \mathrm{~cm}$ with selective inner selective catheter.

2. Which guidewire should I use after groin access? $180 \mathrm{~cm}$ Glidewire advantage (Terumo Medical, Somerset, New Jersey, USA).

3. Should I primarily catheterize the target vessel with a standard diagnostic catheter and use an exchange wire for placing the long sheath, or use an inner catheter within a long sheath primarily? Use a long sheath $(90 \mathrm{~cm}$ Shuttle Sheath; Cook, Bloomington, Indiana, USA) with an inner selective catheter.

4. Which shape of diagnostic catheter should I use? Determined from CTA. For example, type II and III arches may necessitate the use of a reverse curve catheter (Simmons or VTK type).

5. To confirm LVO, should I perform a formal diagnostic angiogram? Whenever possible, no formal angiogram prior to deployment. Just a simple contrast injection with the fluoroscopic image saved.

6. Should I use the power injector for this angiogram? No.

7. Should I connect the typical continuous flushes to the catheters used in these cases? Not initially.

8. What dose of IV heparin should be administered, if any? If patient received IV tPA, no. If not, 3000 units after groin access.

9. Once there is confirmed LVO, should I use primary aspiration or stent-retriever? Stent-retriever.

Table 1 Demographics comparing Group 1 and Group 2 patients

\begin{tabular}{llll}
\hline & Group 1 & Group 2 & p Value \\
\hline $\mathrm{n}$ & 22 & 61 & \\
Age in years, mean (min-max) & $74.2(37-93)$ & $72.7(19-98)$ & 0.6997 \\
$\%$ Female & $60 \%$ & $60 \%$ & 0.9995 \\
Admission NIHSS, median (min-max) & $16(8-24)$ & $17(4-32)$ & 0.8176 \\
Symptom onset to angiography & $197(164$ to & $219.5(198$ to & 0.8808 \\
(min), median (95\% CI) & $222)$ & $257)$ & \\
Site of intracranial occlusion & & & \\
$\quad$ M1 segment MCA & $77 \%(17 / 22)$ & $74 \%(45 / 61)$ & 0.99 \\
$\quad$ ICA & $23 \%(5 / 22)$ & $26 \%(16 / 61)$ & \\
Cervical ICA stenosis or occlusion & $14 \%(3 / 22)$ & $15 \%(9 / 61)$ & 0.99 \\
Treated with IV tPA & $77 \%(17 / 22)$ & $69 \%(42 / 61))$ & 0.4552 \\
\hline ICA, internal carotid artery; MCA, middle cerebral artery; NIHSS, National Institute of \\
Health Stroke Scale; tPA, tissue plasminogen activator. & & \\
\hline
\end{tabular}

10. If I'm using a stent-retriever, should I use local aspiration or a balloon guide catheter? Local aspiration.

11. Which aspiration catheter should I use? We standardized on a single catheter (ACE 64; Penumbra, Almeida, California, USA)

12. Which stent-retriever should I use? Determined from CTA based on location of LVO and vessel size. For example, we generally use $6 \mathrm{~mm}$ devices for larger internal carotid artery and proximal M1 lesions or $4 \mathrm{~mm}$ devices for smaller middle cerebral artery lesions.

13. Which microcatheter and micro-guidewire? Microcatheter determined by answer to \#12 above. We standardized on a single micro-guidewire (Synchro2; Stryker Neurovascular, Kalamazoo, Michigan, USA).

We reduced the number of decisions the neuro-interventional radiology had to make from 13 to 3 (numbers 1,4 , and 12). In addition, the answers to these three can be made on the pretreatment CTA, prior to starting the procedure. The appropriate devices can be pulled and ready prior to groin puncture. Patients treated under this streamlined process represented group 2 (post-standardization).

This process had other implications for the technologists and nurses who work with us:

- We keep the room in an 'ELVO ready' state at all times. The necessary equipment is left sterile, unopened on a table. Once a case is accepted, the technologist should not have to leave the room to obtain equipment needed in the vast majority of cases. This allows for very rapid preparation of the tray and procedure room.

- There is less need for communication (and, thus, less opportunity for miscommunication) during the procedure. The technologists and nurse can concentrate on following the 'routine' protocol.

- As the tray is prepared, the local aspiration catheter, the microcatheter needed for the chosen stent-retriever, and the micro-guidewire are prepared as a unit, flushed and kept ready to use. The stent-retriever is not opened until confirmation of occlusion.

\section{Data collection and statistical methods}

Data were collected from a quality assurance database of all ELVO treatments performed at our institution. The database was searched for the following: patient age, gender, site of intracranial location, admission National Institute of Health Stroke Scale (NIHSS) score, time from symptom onset to angiography, presence of concomitant ipsilateral carotid stenosis or occlusion, pretreatment with IV tPA, time from groin puncture to first deployment of device, time from groin puncture to final recanalization, final recanalization class (based on Modified Thrombolysis in Cerebral Ischemia (mTICI) score), posttreatment symptomatic hemorrhage (defined as new hemorrhage on imaging with at least a 4-point increase in NIHSS), NIHSS at discharge, and in-hospital mortality.

All analyses were conducted using SAS Software V.9.4 (SAS, Cary, North Carolina, USA). Differences between baseline characteristics were assessed using a Wilcoxon test for continuous measures and $\chi^{2}$ or Fisher exact test (two-tailed) for categorical measures using the FREQ and NPAR1WAY procedures. For thoroughness, workflow time was examined using both a generalized linear model assuming a negative binomial distribution (to calculated mean time) and Kaplan-Meier estimation (to calculated median time) using the GLIMMIX and LIFETEST procedures. Significance was established at the 0.05 level and all interval estimates were calculated for $95 \%$ confidence. 

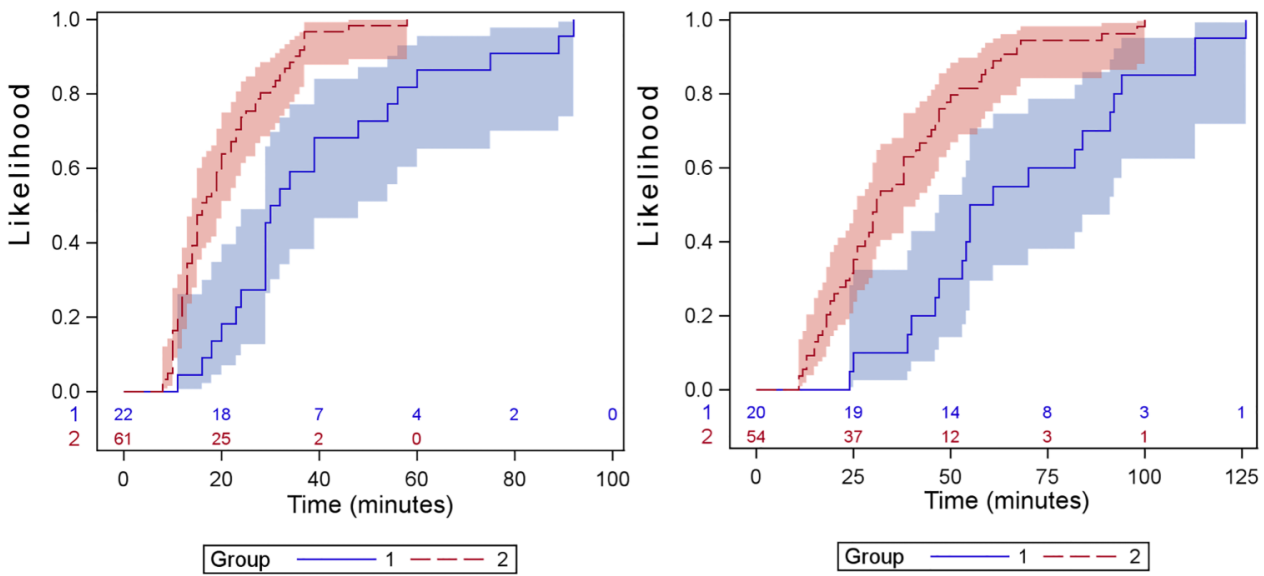

Figure 1 The left panel shows the empirical curve of time between groin puncture and first deployment before and after standardization. The right panel shows the empirical curve of time groin puncture and recanalization (Modified Thrombolysis in Cerebral Ischemia (mTICl) $2 \mathrm{~b}$ or 3 ) before and after standardization (groups 1 and 2, respectively). Both panels include 95\% confidence limits and number of patients left. As can be seen in both panels, the time in group 2 was greatly reduced relative to group 1.

\section{RESULTS}

As indicated in table 1 , no baseline differences were found between those receiving treatment prior to standardization $(n=22)$ and those undergoing the standardized process $(n=61)$. A significant reduction in workflow time was observed after standardization. Mean time from groin puncture to first stent-retriever deployment was $39.8 \mathrm{~min}$ (95\% CI 32.5 to 48.7 ) before standardization and $20 \mathrm{~min}$ (95\% CI 17.6 to 22.8 ) after standardization, a relative reduction of $49.7 \% \quad(\mathrm{p}<0.0001)$. Median time from groin puncture to first deployment also reduced significantly from $31 \mathrm{~min}(95 \%$ CI 29 to 48$)$ to $16 \mathrm{~min}$ (95\% CI 14 to 20$),(\mathrm{p}<0.0001$; see figure 1$)$.

The mean time from groin puncture to final recanalization was $68.2 \mathrm{~min}$ (95\% CI 54.4 to 85.4 ) before standardization and $37 \mathrm{~min}(95 \% \mathrm{CI} 32.1$ to 42.5$)$ after standardization, a relative reduction of $45 \%(\mathrm{p}<0.0001)$. Median time from groin puncture to final recanalization was $58 \mathrm{~min}(95 \% \mathrm{CI} 47$ to 91$)$ for group 1 and $31 \mathrm{~min}(95 \%$ CI 26 to 41$)$ for group 2 $(\mathrm{p}<0.0001$; see figure 2$)$.

There were no differences in recanalization rate, and no significant intraprocedural complications despite a more rapid approach to the procedure (table 2). An example of extremely rapid recanalization is shown in figure 1 .

\section{DISCUSSION}

As the embolectomy era matures, we will all continue to improve our times to recanalization. Clearly the time decay curve for patients with ELVO is steep, and we felt that every possible chance to improve times to treatment is valuable. Previous efforts to optimize pre-angiography workflow have shown decreases in time to treatment, but little has been written on the benefits of a standardized approach to the patient once the case begins. We feel there are many benefits to this approach.

First, every member of the team is familiar with the steps. There is no need to wonder which neurointerventionalist is on call and how they wish to treat the patient. For the treating neurointerventionalist there are fewer steps on which to make decisions. This serves to decrease the 'cognitive load' during a stressful situation, thus allowing neurointerventionalists to focus their cognitive resources in other areas which can result in faster decision-making.

Second, there is a benefit to all the members of the team. Trainees who may be scrubbing in are more able to assist earlier in their education since the setup is standardized. The technologists and nurses have fewer questions to ask, potentially allowing the neurointerventionalist to concentrate on the task at hand.
A

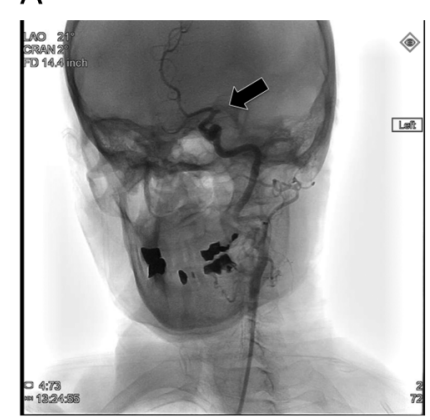

B

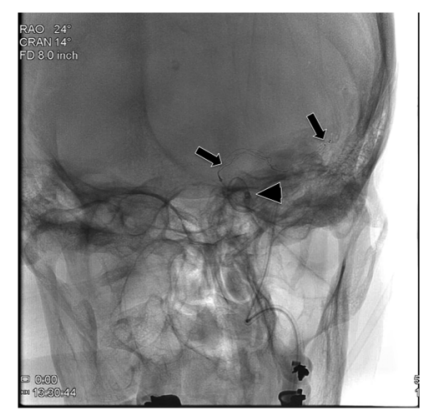

C

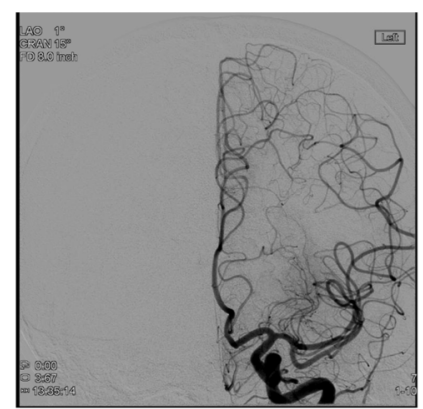

Figure 2 An example of extremely rapid recanalization as a result of a standardized approach. This patient presented with a left M1 segment middle cerebral artery occlusion (arrow, A) and an admission National Institute of Health Stroke Scale (NIHSS) score of 25. The standardized approach was used with a stent-retriever placed across the occlusion (arrows, B) along with the distal aspiration catheter (arrowhead, B). After retrieval, Thrombolysis in Cerebral Ischemia (TICl) 2 b flow was restored (C) with a single distal branch occlusion (not shown). Discharge NIHSS score was 1. Note the timestamps on the images which show the actual times, and groin puncture was 2 min prior to the first image in (A). Total time from groin puncture to first deployment was $8 \mathrm{~min}$ and from groin puncture to final recanalization was $13 \mathrm{~min}$. 
Table 2 Procedural workflow characteristics and clinical follow-up

\begin{tabular}{|c|c|c|c|}
\hline & Group 1 & Group 2 & p Value \\
\hline $\begin{array}{l}\text { Groin puncture to first deployment } \\
(\min ) \text {, mean }(95 \% \mathrm{Cl})\end{array}$ & $\begin{array}{l}39.8 \text { (32.5 to } \\
48.7)\end{array}$ & $\begin{array}{l}20.0(17.6 \text { to } \\
22.8)\end{array}$ & $<0.0001$ \\
\hline $\begin{array}{l}\text { Groin puncture to final recanalization } \\
\text { (min), mean }(95 \% \mathrm{Cl})\end{array}$ & $\begin{array}{l}68.2 \text { (54.4 to } \\
85.4)\end{array}$ & $\begin{array}{l}37.0 \text { (32.1 to } \\
42.5)\end{array}$ & $<0.0001$ \\
\hline \multicolumn{4}{|l|}{ Final recanalization } \\
\hline $\mathrm{TICl} 2 \mathrm{~b} / 3$ & $77 \%(17 / 22)$ & $82 \%(50 / 61)$ & 0.85 \\
\hline $\mathrm{TICl} 2 \mathrm{a}$ & $14 \%(3 / 22)$ & $7 \%(4 / 61)$ & \\
\hline $\mathrm{TICl} 0 / 1$ & $9 \%(2 / 22)$ & $11 \%(7 / 61)$ & \\
\hline Symptomatic hemorrhage & $10 \%$ & $4 \%$ & NS \\
\hline $\begin{array}{l}\text { NIHSS at discharge, median (min- } \\
\text { max) }\end{array}$ & $4(0-20)$ & $7(0-30)$ & 0.4149 \\
\hline \% in-hospital mortality & $14 \%(3 / 22)$ & $15 \%(9 / 61)$ & 0.99 \\
\hline
\end{tabular}

This may be even more critical when procedures are performed after hours with staff that may be more tired. In the future we expect continued decreases in recanalization times as familiarity with the process grows.

There are some disadvantages to this approach. It is certainly possible that there are certain circumstances where alternate approaches may be preferable. For example, patients with tandem occlusions of the extracranial internal carotid artery (ICA) along with an intracranial occlusion may need angioplasty and/or stenting of the ICA before access to the intracranial embolus can be achieved. In these cases, the equipment may need to vary. Physicians may also have individual biases towards alternate techniques (eg, balloon guide vs local aspiration; or stent-retriever-based vs primary aspiration alone). This approach does remove some individual variability from the hands of the physician. There is also the disadvantage that we did not show a difference in outcomes in this small study, but that might be because of the small sample size. Further studies with larger cohorts may show a benefit in outcomes.
Ultimately the patient benefits as faster recanalization results in improved outcomes, and we feel this is a simple step that every institution can incorporate into their practices.

\section{CONCLUSION}

A standardized approach to the equipment used in ELVO thrombectomy cases across an institution may result in dramatically reduced times to recanalization.

Contributors All authors were responsible for substantial contributions to the conception or design of the work; or the acquisition, analysis, or interpretation of data for the work; and drafting the work or revising it critically for important intellectual content; and final approval of the version to be published; and agreement to be accountable for all aspects of the work in ensuring that questions related to the accuracy or integrity of any part of the work are appropriately investigated and resolved.

Competing interests None declared.

Provenance and peer review Not commissioned; externally peer reviewed.

\section{REFERENCES}

1 Berkhemer OA, Fransen PS, Beumer D, et al. A randomized trial of intraarterial treatment for acute ischemic stroke. N Engl J Med 2015;372:11-20.

2 Goyal M, Demchuk AM, Menon BK, et al. Randomized assessment of rapid endovascular treatment of ischemic stroke. N Engl J Med 2015:372:1019-30.

3 Campbell BC, Mitchell PJ, Kleinig TJ, et al. Endovascular therapy for ischemic stroke with perfusion-imaging selection. N Engl J Med 2015:372:1009-18.

4 Jovin TG, Chamorro A, Cobo $E$, et al. Thrombectomy within 8 hours after symptom onset in ischemic stroke. N Engl J Med 2015;372:2296-306.

5 Saver JL, Goyal M, Bonafe A, et al. Stent-retriever thrombectomy after intravenous t-PA vs. t-PA alone in stroke. N Engl J Med 2015;372:2285-95.

6 Khatri P, Abruzzo T, Yeatts SD, et al. Good clinical outcome after ischemic stroke with successful revascularization is time-dependent. Neurology 2009;73:1066-72.

7 Fransen PS, Berkhemer OA, Lingsma HF, et al. Time to reperfusion and treatment effect for acute ischemic stroke: a randomized clinical trial. JAMA Neurol 2016;73:190-6.

8 Rai AT, Smith MS, Boo S, et al. The 'pit-crew' model for improving door-to-needle times in endovascular stroke therapy: a Six-Sigma project. J Neurointerv Surg 2016:8:447-52.

9 Aghaebrahim A, Streib C, Rangaraju S, et al. Streamlining door to recanalization processes in endovascular stroke therapy. J Neurointerv Surg. Published Online First: 5 Apr 2016.

10 Sweller J. Cognitive load during problem solving: effects on learning. Cogn Sci 1988; 12:257-85. 